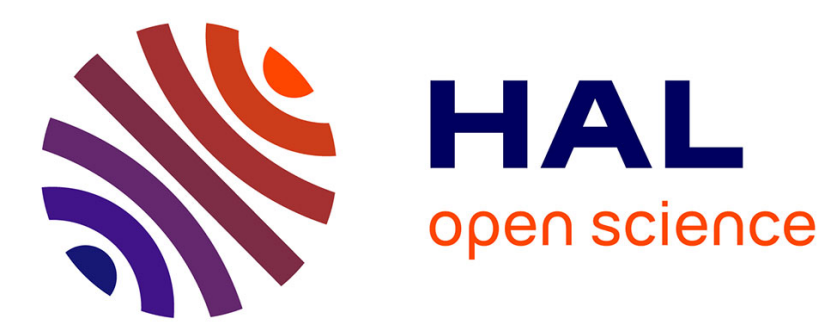

\title{
ON THE SEARCH FOR EXPERIMENTALLY OBSERVED GRAIN BOUNDARY PHASE TRANSITIONS
}

R. Balluffi, T. Hsieh

\section{- To cite this version:}

R. Balluffi, T. Hsieh. ON THE SEARCH FOR EXPERIMENTALLY OBSERVED GRAIN BOUNDARY PHASE TRANSITIONS. Journal de Physique Colloques, 1988, 49 (C5), pp.C5-337-C5-349. 10.1051/jphyscol:1988540 . jpa-00228037

\section{HAL Id: jpa-00228037 https://hal.science/jpa-00228037}

Submitted on 1 Jan 1988

HAL is a multi-disciplinary open access archive for the deposit and dissemination of scientific research documents, whether they are published or not. The documents may come from teaching and research institutions in France or abroad, or from public or private research centers.
L'archive ouverte pluridisciplinaire HAL, est destinée au dépôt et à la diffusion de documents scientifiques de niveau recherche, publiés ou non, émanant des établissements d'enseignement et de recherche français ou étrangers, des laboratoires publics ou privés. 
ON THE SEARCH FOR EXPERIMENTALLY OBSERVED GRAIN BOUNDARY PHASE TRANSITIONS

\section{R.W. BALLUFFI and T.E. HSIEH}

Department of Materials Science and Engineering, Massachusetts Institute of Technology, Cambridge, MA 02139, U.S.A.

Abstract - The phase space for a heterogeneous system containing a grain boundary involves a relatively large number of variables (i.e., at least six plus the number of components), and it is therefore conceptually possible to induce a large variety of grain boundary phase transitions by selectively varying these parameters. Despite this, a review of the 1iterature reveals that there have been virtually no clear-cut experimental observations of txansitions reported in which the boundary structure has been observed as a function of time under well defined conditions. In current work, we are searching for roughening/faceting transitions and melting transitions for boundaries in Al by hot stage transmission electron microscopy. A clear example of a reversible roughening/faceting transition has been found. No evidence for melting has been found for temperatures as hîgh as $0.96 \mathrm{~T}_{\mathrm{m}}$ (by monitoring GBD core delocalization in several special boundaries with $\Sigma \leq 13$ ) or $0.999 \mathrm{~T}_{\mathrm{m}}$ (by observing the local diffraction contrast at general boundaries in polycrystalline specimens).

1. Introduction - Since grain boundaries may be regarded as phases, the local equilibrium of regions containing boundaries may be treated using the standard methods of the heterogeneous equilibrium of multi-phase systems. However, as discussed by Cahn (1), the phase space becomes relatively complicated since it is then necessary to introduce additional state variables in order to specify the boundary geometry. These variables include the three required to specify the crystal misorientation $\left(R_{1}, R_{2}, R_{3}\right)$ and the two required to specify the inclination of the boundary plane $\left(\mathrm{N}_{1}, \mathrm{~N}_{2}\right)$. Under many conditions a boundary will automatically relax to its equilibrium translational state when the above five variables are fixed, and they are then. sufficient to specify the boundary geometry. In the following, we shall assume, with Cahn (1), that this is the case. It therefore follows that the number of variables required for a heterogeneous system containing a boundary is $6+C$, where $C$ is the number of components. A useful choice is $R_{1}, R_{2}, R_{3}, N_{1}, N_{2}, T, P, X_{1}, X_{2} \ldots X_{C-1}$ where $T=$ temperature, $P=$ pressure, and the $\mathrm{X}_{i}$ are compositions. A patch of boundary corresponding to a given point in

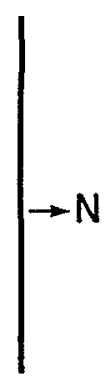

(a)

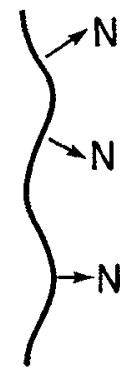

(b)

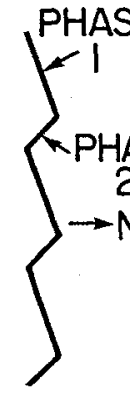

(c)

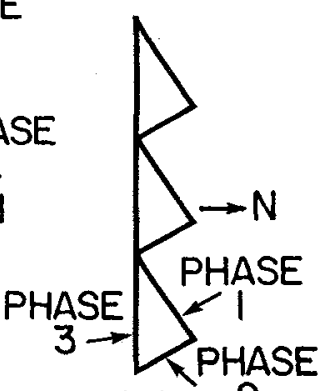

(d) phase space may consist of one boundary phase (if, for example, it belongs to a boundary which is flat or smoothly curved as in Fig. 1a or 1b) or more than one phase (if, for example, it is faceted as in Fig. lc or dissociated as in Fig. 1d). Single phase and multiphase regions therefore exist, and appropriate phase diagrams may be constructed (1).

In view of the above, it seems likely that a rich variety of grain boundary phase transitions should occur over 
different regions of phase space when the state variables are varied. this expectation, there have been virtually no clear-cut experimental observations of such transitions reported in the literature where the structure has been observed at various stages of the transition under well defined conditions. In the following, we first give a brief review of some of the previous experimental work relevant to this point and then describe some current results from our laboratory in wich we have searched for roughening, faceting and melting transitions under better defined conditions for boundaries in A1 by hot stage transmission electron microscopy (TEM). The focus of the present paper is on experimental results, and little effort is made to discuss attempts to calculate boundary phase transitions.

\section{Brief Review of Some Previous Experimental Results}

Numerous reports exist in which a discontinuity of some type has been found in some process associated with grain boundaries. It has often been suggested (e.g., citations in References 1-3 and also 4) that such discontinuities are due to boundary phase transitions. However, no direct evidence of this has been presented and they therefore remain unconvincing.

Evidence for faceting transitions of the type [one-phase/flat boundary] $\rightarrow$ [two-phase/faceted boundary] driven by changes in composition has been found in $(5,6)$. Bishop, Hartt and Bruggeman (5) found that the faceting of tilt boundaries in zinc tended to fade out in regions of bicrystal specimens where relatively high concentrations of solute atoms were expected to be present. However, no actual concentrations were measured. Donald (6) found that the addition of small concentrations of $\mathrm{Bi}$ to polycrystalline $\mathrm{Cu}$ caused a high incidence of boundary faceting. Unfortunately, the behavior of specific boundaries, with and without $\mathrm{Bi}$, could not be studied in this work.

Dissociated grain boundary structures of the type illustrated in Fig. 1d have been observed, e.g. $(7,8)$, but actual transitions from, say, a [onephase/flat boundary] to such a [three-phase/dissociated boundary] have not.

Sickafus and Sass (9) reported that patches of two different boundary structures (phases) coexisted in flat areas of small angle [001] twist boundaries in $\mathrm{Fe}$ containing certain $\mathrm{Au}$ solute atom concentrations. Furthermore, the ratio of the amounts of the two phases changed as the apparent solute concentration changed, and they took this as evidence of a boundary phase transition of the type [one-phase/flat boundary] $\rightarrow$ [two-phase/flat boundary] driven by a change in composition. However, it was known that the distribution of Au atoms was not equilibrated throughout the specimens and, furthermore, the actual concentrations were not measured locally. Also, the relative amounts of the two phases did not always vary systematically with the apparent Au concentration as expected in a two-phase mixture. Further work seems called for. 
Glicksman and Vold (10) reported an apparent discontinuity in the dihedral angle produced by the surface tension of symmetric tilt boundaries in $B I$ at the liquid/solid interface as the tilt angle, $\theta$, was increased as shown in Fig. 4 of (10). This was interpreted as a first order congruent phase transition of the type [solid phase/flat boundary] $\rightarrow$ [1iquid phase/flat boundary] driven by the change in $\theta$ at a temperature very close to the bulk melting temperature, $T_{m}$. However, as discussed by Rottmann (11), such a discontinuity does not seem plausible thermodynamically, and further work is therefore called for.

- Many efforts, summarized in (12), have been made to determine whether grain boundaries (including both general boundaries and particular grain boundaries) undergo congruent melting [1]* before bulk lattice melting upon raising the temperature to $T_{m}$. Most of those experiments using bulk specimens have ylelded ambiguous results (12), but Glicksman and Vold $(13,14)$ used hot stage TEM to observe directly the structure of the grain boundaries present in nominally 99.9998 pure polycrystalline Bi films which were directly adjacent to regions which were completely melted. Under these conditions, a temperature gradient existed in a direction normal to the 1 iquid/solid interface, and grain boundaries adjacent to the interface could be observed at temperatures only very slightly below $T_{m}$. Narrow melted regions along many of these grain boundaries (particularly at triple junctions) were observed indicating grain boundary melting at temperatures only very slightly below $\mathrm{T}_{\mathrm{m}}$. However, no information was provided regarding: (a) the magnitudes of the temperature differences which existed; (b) the possible presence of impurities which may have affected the observations; and (c) the degree to which the specimens were equilibrated.

Chan, Liu and Balluffi (3) used hot stage TEM to study the behavior of grain boundary dislocations (GBDs) in near- 55 [001] twist and [001] symmetric tilt boundaries in Al during heating to near $\mathrm{T}_{\mathrm{m}}$. The GBDs were still clearly visible via dislocation-type diffraction contrast at temperatures as high as $0.89 \mathrm{~T}_{\mathrm{m}}$ (for the twists) and $0.92 \mathrm{~T}_{\mathrm{m}}$ (for the tilts). This result indicates that the boundaries retained significant order to these temperatures and had not melted, since melting would have caused a delocalization of the GBDs.

$[1]^{*}$ We take a "melted" boundary to be a boundary which consists of a layer of material having a structure similar to that of a liquid. We do not consider as "melted" a boundary which is undergoing the low temperature structural transitions which may precede complete melting in the manner described by Kikuchi and Cahn (23). (See our further discussion of this point in Section 4.) 

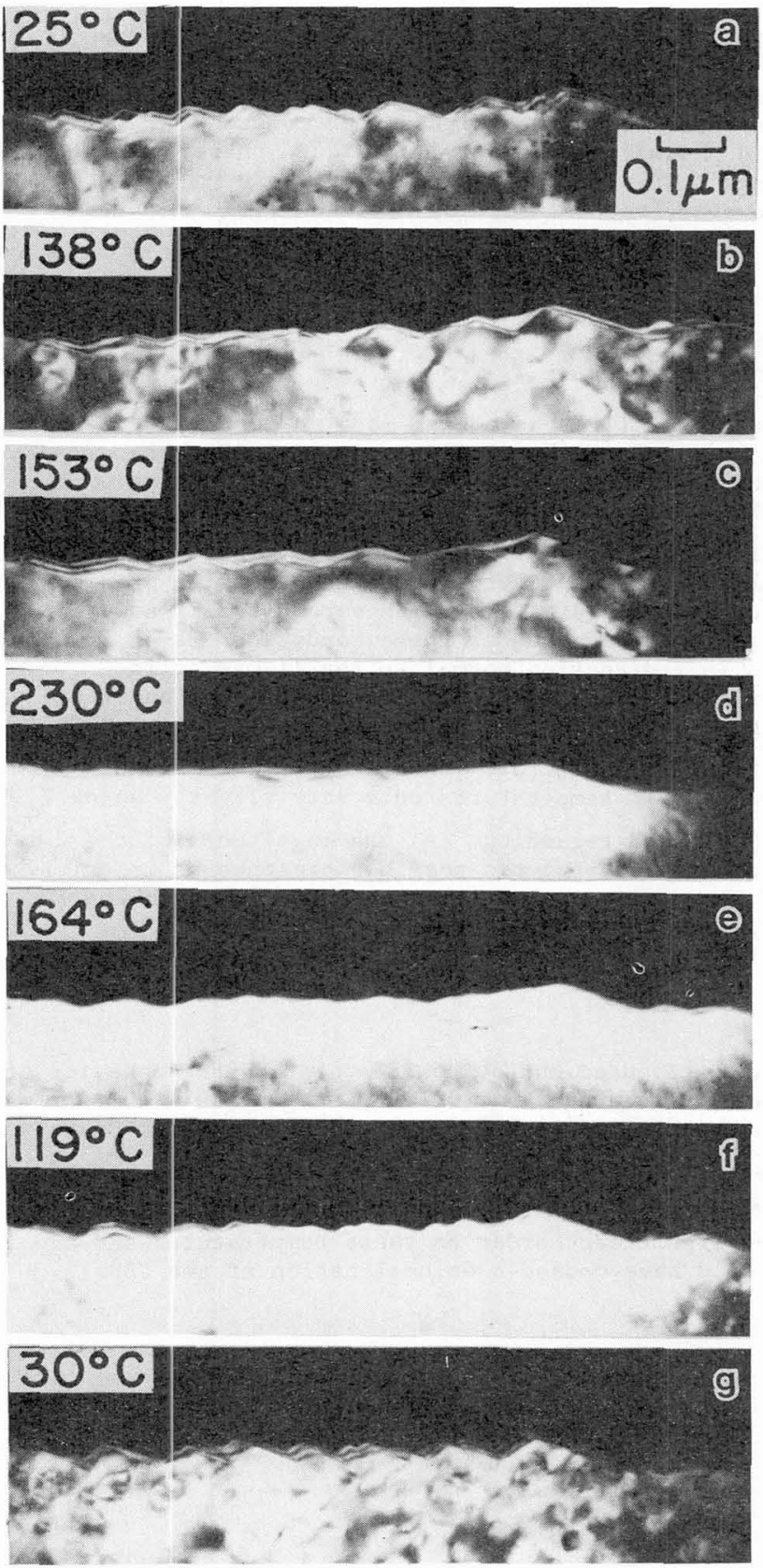

Fig. 2. Structure of $\Sigma 3<111>$ tilt boundary in Al as a function of heating, $(a) \rightarrow(d)$, and subsequent cooling, $(d) \rightarrow(g)$. (Unrelaxed atomistic structure shown in Fig. 3) Dark field diffraction contrast with lower crystal excited. Time at each temperature $\simeq 40 \mathrm{~min}$.
- Erb and Gleiter (15) sintered a random distribution of single crystal $\mathrm{Cu}$ balls to a single crystal Cu plate and observed the rotations of the balls into misorientations which produced boundaries of relatively low energy in the neck regions between the balls and the plate. It was found that many of the low energy misorientations which were observed persisted to temperatures as. high as $0.98 \mathrm{~T}_{\mathrm{m}}$. This

indicates that the boundaries corresponding to at least these low energy misorientations must have retained significant order and were not melted at this relatively high temperature. Unfortunately, these boundaries were not identified geometrically.

\section{Current Experimenta1} Results

\subsection{Exerimental} procedure - Two main types of Al TEM specimens containing grain boundaries were prepared using material of initial 99.999 purity.

Polycrystalline thin films ( $400 \mathrm{~nm}$ thick) of grain size $-1 \mu \mathrm{m}$ were prepared by vapor deposition on (100) cleaved $\mathrm{NaCl}$ single crystal surfaces at $-250^{\circ} \mathrm{C}$ in a standard $10^{-7}$ torr bell jar system. In addition, bicrystal thin film specimens $(-400 \mathrm{~nm}$ thick), each containing a single straight grain boundary of controlled geometry running perpendicular to its surface, as illustrated. 
in Fig. 1 of (3), were prepared by epitaxial vapor deposition on specially prepared $\mathrm{NaCl}$ bicrystal substrates at $340^{\circ} \mathrm{C}$. Further details are given in (3). The polycrystalline specimens were then oxidized in air at $500^{\circ} \mathrm{C}$ for $10 \mathrm{~h}$ in order to increase the grain size and grow a moderately thick oxide layer on each surface. These specimens were then clamped between folding Au grids and mounted in the double-tilt hot stage of a JEOL $200 \mathrm{CX}$ microscope for observation during heating and cooling experiments up to the melting point. These specimens had the advantage that they possessed transverse boundary structures which were quite stable against migration, and a stable encapsulating oxide film which: (a) maintained specimen integrity even after patches of the metal were locally melted; and (b) protected the Al films from outside impurity contamination by acting as an effective diffusion barrier (see below). The hot stage and its temperature calibration are described in (3).

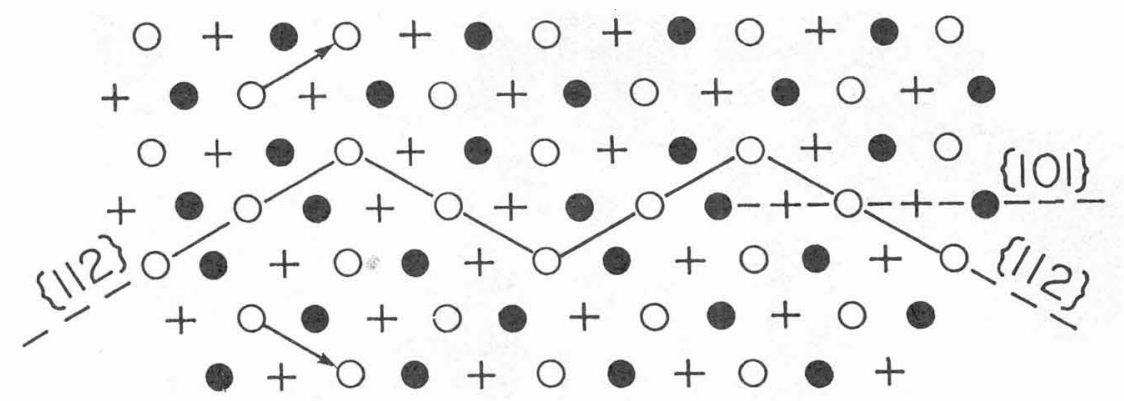

Fig. 3. Unrelaxed structure of faceted $\Sigma 3<111\rangle$ tilt boundary in Al viewed along $\langle 111\rangle$. Different symbols indicate different planes in the $A B C A B C$...stacking sequence.

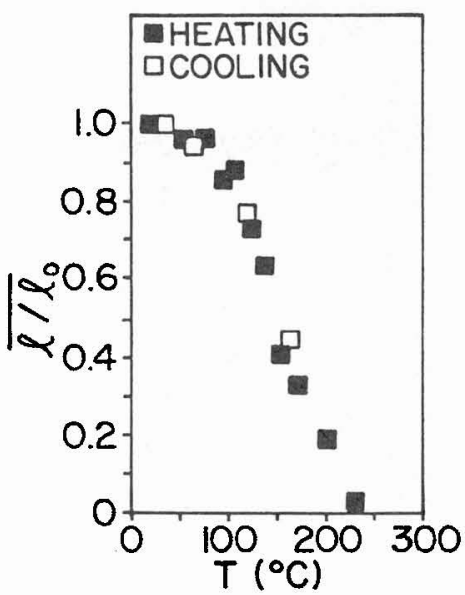

Fig. 4. Average amplitude, $\overline{\ell / \ell}$, of facets on $\Sigma 3<111>$ tilt boundary in Al (shown in Fi.g. 2) as a function of temperature during heating and cooling. Total heating time $\approx 300$ min. Total cooling time $\simeq 150 \mathrm{~min}$.
3.2. Observation of boundary roughening/faceting during heating and cooling - A search of a polycrystalline specimen revealed the boundary shown in Fig. $2 a$ which was identified as a $\Sigma 3<111>$ tilt boundary having an average

inclination along the common $\{101\}$ planes of Crystals 1 and 2 in the $\Sigma 3 \mathrm{CSL}$ and two sets of facets each inclined along common (112) planes of Crystals 1 and 2, as shown in Fig. 3. Upon heating, the faceted structure became progressively rounded as roughening occurred (Fig. 2b) and eventually became essentially flat (Fig. 2d). Upon cooling, the boundary structure reversed its history and eventually became sharply faceted again (Fig. 2g). The time-temperature dependence of the transition was studied by heating in a step-wise fashion. The degree of roughening (as measured by the average amplitude of the facets)

increased rapidly during each incremental temperature increase and then quickly became constant during each subsequent isothermal holding period. This result demonstrated that the degree of roughening was a unique function of 
temperature and was reinforced by the results in Fig. 4 which show the degree of roughening as a function of temperature during both slow heating and cooling. No hysteresis is apparent.

3.3. Observation of melting at grain boundaries during heating and cooling - Polycrystalline specimens were slowly heated to a point where they were partially melted and contained relatively large discrete patches of encapsulated liquid. The temperature was then stabilized, and the boundaries adjacent to the liquid/solid interface were examined under steady state conditions in the manner of Glicksman and Vold $(13,14)$. A typical structure is shown in Fig. 5, and it is seen that many boundaries were wet by the liquid and replaced by narrow layers of liquid. The melted regions were clearly distinguished from the crystalline regions by differences in the local diffraction contrast. The liquid regions generally appeared dark and

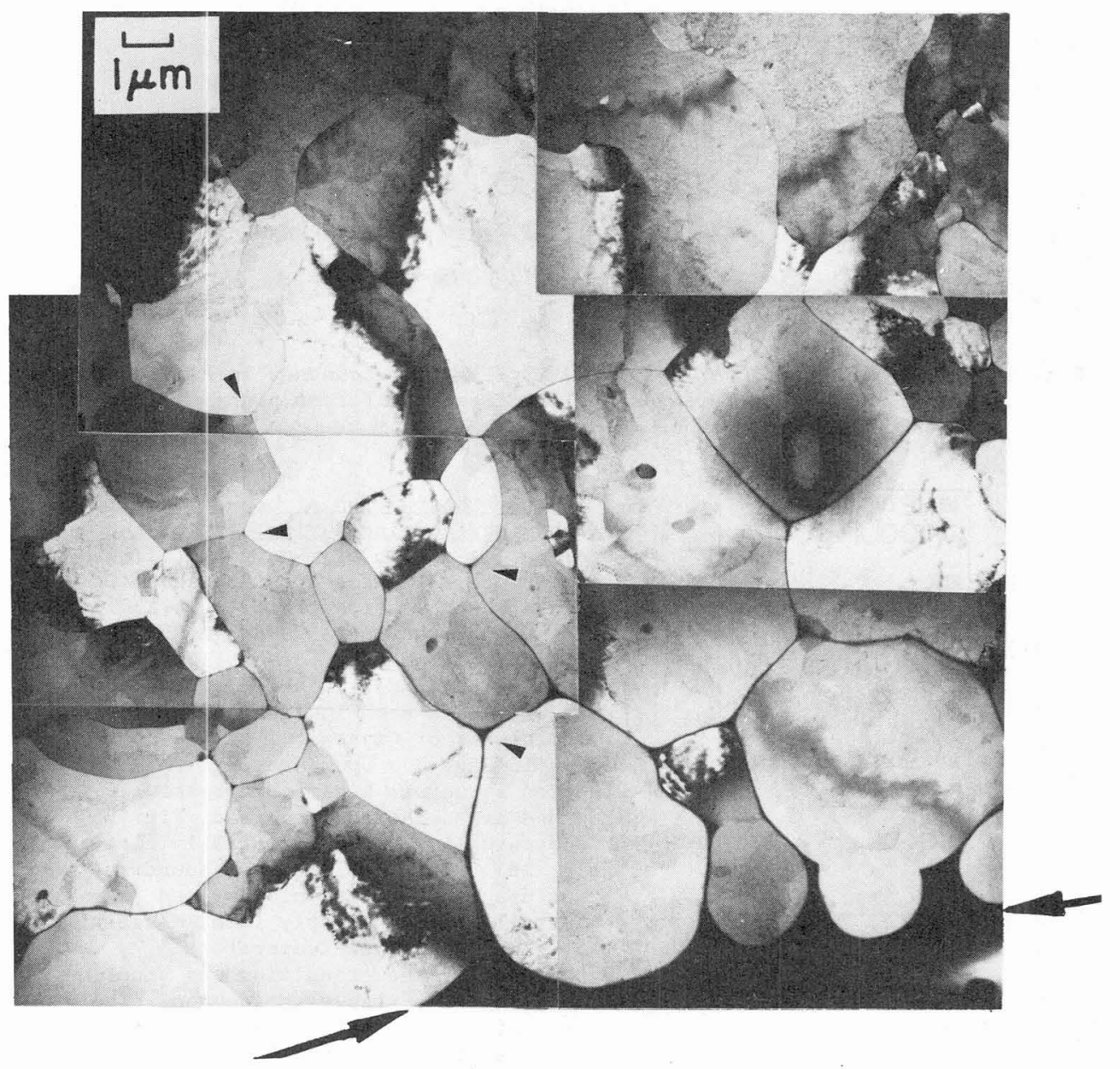

Fig. 5. Structure of polycrystalline Al specimen adjacent to periphery of relatively large melted region (indicated by large arrows). Boundaries near the periphery are wet by the liquid, particularly evident at triple junctions (indicated by sma11 arrows). Structure shown was held -5 min under steady state conditions before being photographed. 

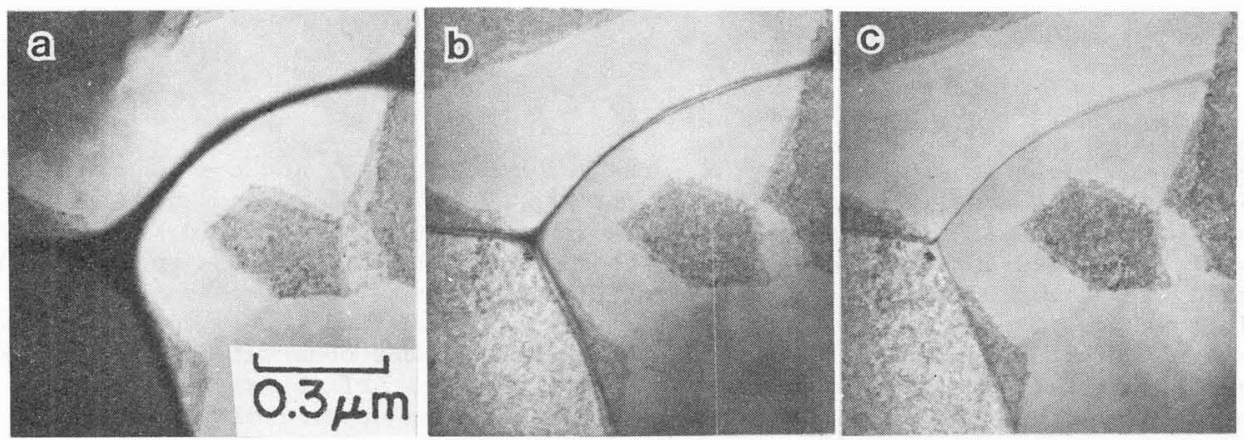

Fig. 6. Disappearance of melted regions at grain boundaries in polycrystalline Al during cooling $\left((a) \rightarrow(c)\right.$ ) from very near $T_{m}$.

exhibited no diffraction contrast, whereas the crystalline regions exhibited the expected strong diffraction contrast. This melting at the grain boundaries was especially evident at triple junctions (see also Fig. 6) and became less extensive as the distance from the liquid/solid interface increased, i.e., as the temperature dropped progressively below $T_{m}$ because of the temperature gradient which existed perpendicular to the interface. Also, no sign of melting was observed at low angle boundaries, i.e., relatively low energy boundaries with structures composed of arrays of discrete lattice dislocations. (No detailed information has yet been obtained which allows us to distinguish structurally between the remaining boundaries which appeared structureless under the microscope and were either associated, or not associated, with a melted layer.) Upon subsequent cooling, the liquid/solid interface moved toward the liquid as expected, and the liquid layers at the melted boundaries progressively disappeared (i.e., crystallized) in an apparently reversible fashion as the local temperature decreased, as seen in Fig. 6 .

Careful examination of micrographs such as Fig. 5 showed that no detectable melting at any grain boundaries (including triple junctions) could be detected at distances from the 1iquid/solid interface $\geq 15 \mu \mathrm{m}$. A conservative estimate of the minimum width of the melted region at a boundary which could be deduced from work with the present specimens using the diffraction contrast method is about $1 \mathrm{~nm}$. This is based on the observation that the melted material was most readily detected at the triple junctions where the width of the pools was measured to be several times as wide as the corresponding melted width at a straight section of boundary (e.g., Fig. 6). The narrowest triple junction images which could be observed were about 2 nm wide, and, hence, the corresponding narrowest melted width at a straight section is $-2 / 3=0.7 \mathrm{~nm}$.

It is of primary importance to estimate an upper limit for the temperature drop, $\Delta \mathrm{T}$, which existed between the temperature at the liquid/solid interface and the temperature at which no melting at the boundaries could be detected. The disc-shaped specimen was clamped around its periphery in the heating stage and heated by inward conduction. On the other hand, the surface lost heat everywhere by radiation causing a cooling of the central region of the specimen. The steady state heat equation for this situation is then 


$$
\frac{\mathrm{d}^{2} \mathrm{~T}}{\mathrm{dr}}+\frac{1}{\mathrm{r}} \frac{\mathrm{dT}}{\mathrm{dr}}=\frac{2 \epsilon\left(5.679 \times 10^{-12}\right) \mathrm{T}^{4}}{\mathrm{kt}}
$$

where $\epsilon=$ hemispherical emissivity, $\mathrm{K}=$ thermal conductivity, and $t=$ specimen thickness. This non-linear equation was integrated numerically, and the results indicated a maximum temperature gradient in the specimen $\approx 450 \mathrm{~K} / \mathrm{cm}$. However, the focused electron microscope beam delivered energy to the specimen locally which tended to heat the specimen interior in a reverse fashion. This effect was estimated using methods similar to those described in (16), and the results indicated a gradient $\leq 240 \mathrm{k} / \mathrm{cm}$ in the region under observation. Since these gradients would tend to oppose each other over most of the specimen, and since the gradient due to radiation losses is a generous upper limit, we may

conclude that $\Delta T$ was certainly $<1^{\circ}$. We therefore conclude that no grain boundary melting was detectable by local diffraction contrast at temperatures $<0.999 \mathrm{~T}_{\mathrm{m}}$.

It is important to point out that impurities may have played at least some role in the very limited amount of boundary melting observed since it is conceivable that low concentrations of impurities were picked up in a manner which could not be detected. For example, we note that concentrations of typical solutes at the $10^{-4}$ level can depress the bulk liquidus temperature by several tenths of a degree. Typical diffusion lengths for substitutional solutes in $A 1$ at $T_{m}$ after the relatively short time of $10^{2} \mathrm{~s}$ are $10-20 \mu \mathrm{m}$, and since the grain size was $-3 \mu \mathrm{m}$, impurity atoms should easily have been able to equilibrate throughout the specimens in the present experiments. In any event, our conclusion that no boundary melting was detected at any boundaries below $0.999 \mathrm{~T}_{\mathrm{m}}$ still stands.

3.4. Observation of localized GBDs at elevated temperatures - In this work, the appearance of localized GBDs in bicrystal specimens was monitored during quasi-equilibrium heating to $T_{m}$ in hot stage TEM experiments similar to those conducted by Chan, Liu and Balluffi (3) described in Section 2 . of central intexest was the extent to which the GBDs remained localized during the heating. In the previous work, near- $\Sigma 5$ boundaries were studied, and no evidence for boundary melting was found at temperatures $\leq 0.92 \mathrm{~T}_{\mathrm{m}}$. The question of whether longer period boundaries of higher $\Sigma$ melt at lower temperatures remains, however, and we therefore tested near- $\Sigma 13$ [001] symmetric tilt boundaries running paralle1 to $\{510\}$ planes. These boundaries possessed arrays of edge GBDs with Burgers vector $\vec{b}=a / \sqrt{26}<510>$ accommodating a small tilt misorientation as seen in Fig. 7a. No detectable delocalization of the GBD cores was found upon heating to $0.96 \mathrm{~T}_{\mathrm{m}}$ as seen in $\mathrm{Fig}$. $7 \mathrm{~b}$. We. therefore conclude that $\Sigma 13$ boundaries do not melt at temperatures $\leq 0.96 \mathrm{~T}_{\mathrm{m}}$.
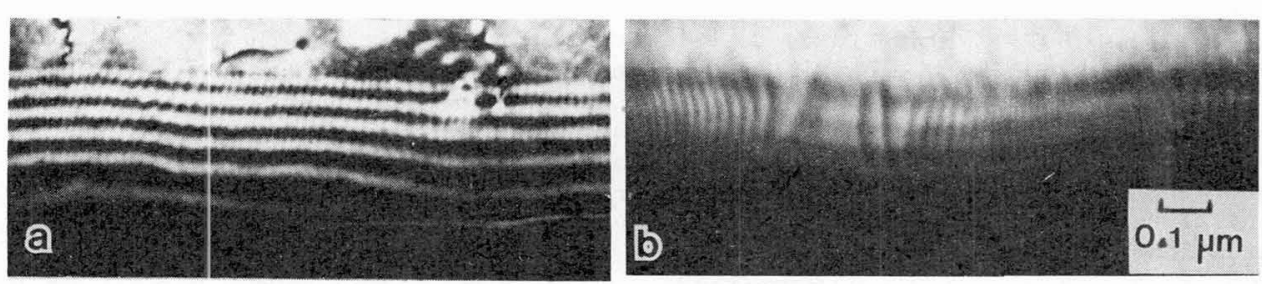

Fig. 7. Localized GBDs observed in near $-\Sigma 13$ [001] symmetric tilt boundary running parallel to $\{510\}$ planes in $\mathrm{Al}$. (a) at $25^{\circ} \mathrm{C}$. (b) at $0.96 \mathrm{~T}_{\mathrm{m}}$. 


\section{Concluding Remarks}

The roughening/faceting boundary transition which we have observed proceeded just as would be expected on the basis of analogous roughening/faceting transitions on free surfaces where sharply faceted structures are stable at low temperatures and where rounding of the edges occurs and more isotropic shapes become stable as the temperature is raised (17). As Cahn has pointed out (1), faceting boundary transitions may generally be expected to be relatively common types of transitions.

Our results that no evidence for melting could be detected at temperatures as high as $0.96 \mathrm{~T}_{\mathrm{m}}$ for several boundaries with $\Sigma \leq 13$ (by the GBD core delocalization technique) or as high as $0.999 \mathbf{T}_{m}$ for general boundaries in polycrystals (by observing the local diffraction contrast) seem mutually consistent. We also remark that these results seem to be consistent with the single crystal ball rotation experiments of Erb and Gleiter (15) discussed in Section 2 .

With respect to calculations, we note that the experimental results described here appear to be inconsistent with calculations reported in (18-20) and apparently consistent with those reported in (21-23). In (18-20) boundaries were found to melt suddenly by first order transitions at temperatures well below $\mathrm{T}_{\mathrm{m}}$. In $(21-23)$ boundaries were found to undergo a continuous transition which first became perceptible at relatively low temperatures. This disordering progressed steadily as the temperature was raised, and, at least in (23), culminated finally with complete boundary melting which occurred rather abruptly at $\mathrm{T}_{\mathrm{m}}$. (For example, in (23) the boundary entropy diverged as $-\ln \left(T_{m}-T\right)$ near $\left.T_{m}.\right)$ Kikuchi and Cahn (23) pointed out that the low and high temperature phenomena are parts of the same overall process and elected to term the entire process a "melting transition". In our opinion, it might be appropriate to consider the low temperature phenomena as "premelting". Needless to say, the present experiments could not have detected any such premelting.

More effort is required at this point to extend this work over a wider range of specific boundary types, to approach $T_{m}$ more closely and to evaluate the role of very small impurity concentrations. It would also be useful to employ a more powerful method to study grain boundary disordering on a local atomistic scale such as grain boundary $x$-ray diffraction. For example, this technique might be capable of detecting the disordering associated with the predicted boundary premelting discussed above.

Acknowledgement - Support for this work was provided by the U.S. Department of Energy under Contract DE-FG02-84ER45116.

\section{References}

(1) Cahn, J. W., J. de Physique 43 , Colloque 66, Supple. au $\mathrm{n}^{\mathrm{0}}$ 12, (1982) C6-199.

(2) Hart, E. W., in The Nature and Behavior of Grain Boundaries, p. 155 (1972) Ed. by Hu, H. (Plenum Press, New York).

(3) Chan, S. W., Liu, J. S. and Balluffi, R. W., Scripta Metall. 19, (1985) 1251 .

(4) Watanabe, T., Kimura, S. I. and Karashima, S., Phil. Mag. A 49, (1984) 845.

(5) Bishop, G. H., Hartt, W. H. and Bruggeman, G. A., Acta Metal1. 19, (1971) 37 . 
(6) Donalı, A., Phil. Mag. 34, (1976) 1185.

(7) Goodhew, P. J., Tan, T. Y. and Balluffi, R. W., Acta Metal1. 26, (1978) 557.

(8) Krakow, W. and Smith, D. A. in Grain Boundary Structure and Related Phenomena, Supple. to Trans. Jpn. Inst. Metals 27, (1986) 277.

(9) Sickafus, K. E. and Sass, S. L.; Acta Metall. 35, (1987) 69.

(10) Glicksman, M. E. and Vold, C. I., Surf. Sci. 31, (1972) 50.

(11) Rottman, C., Scripta Metall. 19, (1985) 43.

(12) Gleiter, H. and Chalmers, B., High-Angle Grain Boundaries, p. 113 (1972) (Pergamon Press, New York).

(13) Glicksman, M. E. and Vold, C. L., Acta Metal1. 15, (1967) 1409.

(14) Glicksman, M. E. and Vold, C. L. in Solidification of Metals, ISI Publication 110, p. 37 (1968) (Iron and Steel Institute, London).

(15) Erb, U. and Gleiter, H., Scripta Metall. 13, (1979) 61.

(16) Gale, B., and Hale, K.F., Brit.J.App1.Phys. 12 (1961) 115.

(17) For example: Rottman, C. and Wortis, M., Phys.Rev. B29, (1984) 328.

(18) Kalonji, G., Deymier, P., Najafabadi, R. and Yip, S., Surf.Sci. 144 (1984) 77 .

(19) Deymier, P., Taiwo, A., and Kalonji, G., Acta Metall, in press.

(20) Nguyen, T., Ho, P. S., Kwok, T., Nitta, C. and Yip, S., Phys.Rev.Lett. 57 (1986) 1919 .

(21) Ciccotti, G., Guillope, M., and Pontikis, V., Phys.Rev. B 27 (1983) 5576.

(22) Guillope, M., Ciccotti, G., and Pontikis, V., Surf.Sci. 144 (1984) 67.

(23) Kikuchi, R. and Cahn, J. W., Phys.Rev. B $\underline{21}$ (1980) 1893.

N.W. Ashcroft: Can the encapsulation geometry encompass the possiblity of significant voids in the Al film, so that the question of surface melting might be examined? On a free surface of lead, surface melting has been reported (Franck \& Van der Veem) to begin at a temperature very substantially below the bulk temperature. If a surface melting signature was available in your technique, the difference between grain boundary and surface melting (if it exists) might be demonstratable.

R.W. Balluffi: The encapsulation of a specimen containing suitable voids with clean surfaces would probably not be feasible because they would be unstable at temperatures near $T_{m}$ and quickly disappear or be modified in detrimental ways. Unfortunately, mass transport over distances of several microns becomes very rapid near $\mathrm{T}_{\mathrm{m}}$.

V. Pontikis: comment on N. Ashcroft question. The photon beam channeling experiments by Freuken and Van der Veem were able to show that disonder appears on surfaces above $\mathrm{T}=0.8 \mathrm{~T}_{\mathrm{m}}$, but more evidence exists for a long range order loss. Then the question of surface melting is still controversial. 